\section{Being on the shelf}

\section{Graham Harris}

Ecosystems of the World 27: Continental Shelves. Edited by $H$. Postma and J. J. Zijlstra. Elsevier:1988. Pp.421. Dfl.360, \$189.50.

Continental shelves account for only about seven per cent of the surface area of the world's oceans. That figure belies their importance, however, which is due not only to their proximity to human influences but because about 90 per cent of the world's fishery resources are harvested on shelves. So an understanding of the basis of their productivity and interannual variability is of great practical and economic significance.

The book reviewed here begins with seven chapters giving an overview of the physical, chemical, biological and geological characteristics of shelf systems; the last four present detailed case studies of Arctic, tropical and temperate systems. Generally, the perspective is a global one, but there is a decided bias towards examples taken from northern temperate waters, especially the North Sea. Further, although the editors perhaps felt that any explicit discussion of human influences should be restricted to commercial fishing, the book would have been considerably improved by inclusion of a contribution on coastal eutrophication, 'red tides' and other pollution effects from river discharges and the disposal of wastes at sea.

The most useful chapters are those which provide summaries of the relevant physics and geology, together with those on the flows of energy in pelagic systems and the fluctuations in fish populations and commercial fisheries. The examples given show that shelf systems exhibit much interannual variability, and we get a clear view of the general inability of ecological theory to account either for the overall fisheries yield of shelf waters or for the interannual variability in those yields. Cushing provides a particularly good review of the types of production in shelf systems and the ecological efficiencies observed at various levels in the food chain.

What is not clear, even in well-documented systems such as the North Sea, is the extent to which long-term changes in fisheries yields and catch compositions are due to changes in primary production, changes in energy efficiencies at various levels in the food chain, structural changes related to the physical environment or changes in biological interactions due to fishing activity. Equilibrium 'fixed engine' models will certainly not do. Sharp's excellent chapter on fluctuations in fish populations and fisheries rejects a generalist approach in favour of a discussion of the ecological relations between fish, habitat and climates. He reviews the global ecology of fish populations in continental shelves, and ends by stressing the unpredictability of population interactions in fluctuating environments and calling for more realism on the part of fisheries managers.

The first of the four case studies is a comprehensive review of the shelf ecosystem of the north-east United States. Again, the long-term trends in fish biomass and species composition cannot be clearly attributed to the effects of fishing, to predator-prey interactions or to environmental factors - multiple correlation analysis of 22 species of fin-fish and squid revealed little more than might be expected by chance.

Overall, the reader gets a strong

impression of the large volume of research that has been carried out on shelf systems all over the world. Much descriptive information has been gathered about biogeography, the composition of pelagic and benthic communities, and the physical, chemical and geological basis of the ecosystems. What is singularly lacking is a theoretical basis for generalizations and predictions about the ecological processes and interactions which produce the observed interannual variability. For many purposes, the management of shelf systems requires predictions of the effects of human activities in the face of variability in climate. Although this book was long delayed in production, meaning that there are few references dated after 1984, it clearly reveals the shortcomings of present approaches.

Graham Harris is Chief Research Scientist in the CSIRO Division of Fisheries, GPO Box 1538, Hobart, Tasmania 7001, Australia.

\section{A cooler view}

\author{
V. J. Emery
}

Copper Oxide Superconductors. By Charles P. Poole Jr, Timir Datta and Horacio A. Farach. Wiley: 1988. Pp. 289. $\$ 35, £ 27.85$.

A SUDDEN and dramatic development in any field of activity will surely spawn its own literature, the more so when it captures the public imagination and lures thousands of researchers all around the world. High-temperature superconductivity is no exception.

First to get their books to press were the popularizers who chronicled the 'Woodstock' atmosphere of the 1987 March meeting of the American Physical Society or the race to unravel the structure of the first liquid-nitrogen-temperature superconductor. Now, with the passage of time, books designed for a more involved scientific readership are starting to appear. One of the first is Copper Oxide Superconductors which surveys the results of some 12 months of round-the-clock research, mainly in 1987. The scope is avowedly experimental, although there is some discussion of theories, ideas and explanations "to give coherence". The outcome of this attempt at an instant review of more than one thousand papers is not surprising: although there is much of value, the presentation lacks the depth and perspective of a more considered work. It is a sourcebook rather than a textbook.

The authors come closest to attaining their goal in the sections on preparation, characterization and general structural properties. Many people will find it useful to have quick access to the basic informa- tion and a convenient listing of the original work. Although it could not include a number of recent developments, particularly in the preparation of thin films and single crystals, the book does encompass the early body of knowledge.

But it is much more difficult to give a satisfactory account of the measurement and interpretation of physical properties. These are the stuff of controversy. Early experiments were carried out before sample preparation and characterization were fully under control, and it has taken some time to develop an agreed body of data. Early theories were not worked out in enough detail to allow an unambiguous and universally accepted explanation of what is going on. The evenhanded approach adopted by the authors gives an accurate impression of the very wide range of opinion that has been expressed, but does not give a perspective on the issues or a feeling for what must be done to achieve a consensus.

The book bears the mark of correction without revision: one is often arrested by misleading statements, only to find that some reparation is made towards the end of the chapter. But readers who are prepared to form their own opinions will find here an extensive compilation of data and a charted route to the original literature that will be invaluable.

V. J. Emery is in the Department of Physics, Brookhaven National Laboratory, 20 Pennsylvania Street, Upton, New York 11973, USA.

- Journey into Light: the Life and Science of C.V. Raman by G. Venkataraman has now been published in Britain by Oxford University Press ( $£ 22.50$ ). For review see Nature 338, 685; (1989). In addition, Oxford University Press has published Scientific Papers of C.V. Raman, containing his complete works in six separate volumes. 